\title{
Study on the Promotion of Physical Fitness of Primary and Middle School Students in Tibetan Autonomous Prefecture of Garzê from the Perspective of Physical Education in Schools*
}

\author{
Chenting Zhu \\ Physical Culture Institute \\ Leshan Normal University \\ Leshan, China 614004
}

\author{
Beiguang Guan** \\ Physical Culture Institute \\ Leshan Normal University \\ Leshan, China 614004 \\ **Corresponding Author
}

\author{
Jingtao $\mathrm{Wu}$ \\ Physical Culture Institute \\ Leshan Normal University \\ Leshan, China 614004
}

\begin{abstract}
The physical health of primary and secondary school students has always been the focus of constitution researchers. The improvement of the level of social and economic development, the popularization and use of information technologies and smart devices, etc., are all affecting the development of physical fitness of primary and secondary school students. Primary and secondary school students are individuals at the school stage, the school is not only the main place for learning and living for them, but also an important place for them to conduct physical activities. This paper uses literature data method, field research method and interview method to study the primary and middle school students in Tibetan Autonomous Prefecture of Garzê, aiming at mastering the basic situation of primary and middle schools in Tibetan Autonomous Prefecture of Garzê, summing up the reasons for the decline of physical fitness of Garzê Tibetan primary and middle school students, and proposing countermeasures to promote the healthy development of physical fitness of them.
\end{abstract}

Keywords-school physical education; Garzê Tibetans; primary and middle school students; physical health

\section{INTRODUCTION}

In April 2008, the meeting of Political Bureau of the Central Committee of the CPC discussed and adopted the "Opinions of the CPC Central Committee and the State Council on Strengthening Physical Education of Youngsters to Enhance the Physical Fitness of Young People". This is an important decision made by the Party Central Committee and the State Council to cultivate qualified builders and successors

*Project Source: National Social Science Fund Project Western Project Supported by National Social Science Fund "Study on the Evolution of Constitution of Tibetan and Chiang Adolescents in Sichuan and the Attributions" No.: 17XTY006. of the cause of socialism with Chinese characteristics from the strategic height of the comprehensive implementation of the scientific development concept and the construction of a harmonious socialist society ${ }^{1}$.

As an important part of the development of China's socialism, the development of physical fitness of students in the southwestern regions especially the minority nationality regions plays a vital role. The ninth chapter of the "Outline of the National Medium- and Long-Term Education Reform and Development Plan" (2010-2020) (hereinafter referred to as the Outline) clearly states that the national education cause should be attached importance to and supported and the special difficulties and outstanding problems faced by the minority nationality areas in the education undertakings should be effectively solved ${ }^{2}$, the Tibetan Autonomous Prefecture of Garzê as a representative high-altitude national living area in Sichuan, its educational conditions are relatively backward, and close attention should be paid to its education issues.

Combining with the 2017 National Social Science Fund Project Western Project Supported by National Social Science Fund "Study on the Evolution of Constitution of Tibetan and Chiang Adolescents in Sichuan and its Attributions", we conducted investigations, interviews and observations on the physique of students and the school physical education in the Tibetan Autonomous Prefecture of Garzê. In combination with

Bian Siming. Discussions on Promoting the Development of Chiang Students' Physical Health [J].Journal of Aba Teachers University.2008(25)

Bama amo. The status quo, dilemma and breakthrough of the development of education in Tibetan areas in the past ten years --- Taking the Tibetan Autonomous Prefecture of Garzê as an example[J].Journal of Southwest University(Social Science Edition).2012.(1)10. 
the "National Student Physical Health Standards", we have conducted research on the promotion of physical health of Garzê Tibetan students from the geographical environment, education policy and school physical education development of Tibetan Autonomous Prefecture of Garzê.

\section{THE BASIC SITUATION}

\section{A. Natural Environment of Tibetan Autonomous Prefecture of $\operatorname{Garz} \hat{E}$}

Garzê Prefecture is located in the western part of Sichuan Province. It is located in the zone of transition from the first step of China's terrain to the second step of the YunnanGuizhou Plateau and the Sichuan Basin. The crossing zone is located at $27^{\circ} 58^{\prime \prime}$ to $34^{\circ} 20^{\prime \prime}$ north latitude and $97^{\circ} 22^{\prime \prime}$ to $102^{\circ} 29^{\prime \prime}$ east longitude. Garzê Prefecture has a unique geographical position and is part of the Qinghai-Tibet Plateau in the western Sichuan Alpine plateaus of the northern section of the Hengduan Mountains. The terrain slopes from the northwest to the southeast. It is high in the north and low in the south. It is raised in the middle and deep in the southeast. The average elevation of the ground is 3,500 meters. The area belongs to the Qinghai-Tibet Plateau climate, which is characterized by low temperatures, long winters, low precipitation, long sunshine and large temperature differences.

\section{B. "Two Exemptions and One Subsidy" Policy}

The "two exemptions and one subsidy" policy began in 2001. It was originally an education subsidy policy of tuition waiver, book fee waiver, and subsidy of living expenses for boarding students which is aimed at the poor rural students at the compulsory education stage. Since 2006, all the western students have been exempted from tuition and fees, and the central government has also allocated public funds for subsidies. Through this way, the basic cost of schooling and basic living security for primary and middle school students in the western regions have been resolved ${ }^{3}$. The policy has reduced the dropout rate of primary and secondary school students and provided a solid guarantee for the control of dropout.

\section{Physical Health Status of Primary and Middle School Students in Tibetan Autonomous Prefecture of Garz $\hat{E}$}

From the results of many national student physique health surveys since 1979, students' nutrition is guaranteed, and their height, chest circumference and weight have increased. Among them, weight gain is the fastest. The main performance is the significant increase in myopia rate among primary and secondary school students. The number of obesity $(\mathrm{BMI} \geq 28)$ has increased ${ }^{4}$, and the average BMI index of students aged 8 and $10-13$ is significantly lower than the average of the national BMI index, and the physical fitness of students shows a downward trend. There was no significant difference in the

Ye Yingfang. Interpretation of the "two exemptions and one subsidy" policy [J]. Journal of Beijing City University. 2007 (1).

Bian Siming. Discussions on Promoting the Development of Chiang Students' Physical Health [J].Journal of Aba Teachers University.2008(25). vital capacity of students aged 9-11 compared with those of the same age group $(\mathrm{P}>0.05)$. In terms of aerobic endurance, the average value of male students aged 13-15 was 12-20 seconds lower than the average value of male students of the same age in China; the average value of girls aged 13-15 is 16-23 seconds lower than that of girls of the same age in the country, where there were significant differences 5 .

\section{ANALYSIS OF THE REASONS FOR THE DECLINE OF PhysicAl FitNess OF GARZÊ TIBETAN PRIMARY AND MIDDLE SCHOOL STUDENTS}

The development of students' physical health status is constrained by many factors. Since the implementation of the policy of "two exemptions and one subsidy" (exemption of book fees, exemption of tuition and subsidy of living expenses for boarding students) in 2007, the nutritional status of primary and middle school students in Tibetan Autonomous Prefecture of Garzê has basically been guaranteed. Schools in some areas purchase goods in a unified manner, provide students with free meals and milk, etc. as a nutrient supply during the students' study period. The "two exemptions and one subsidy" policy has largely reached the goal of controlling dropping out and guaranteeing students' study, making more students return to school to study. Physical exercise as another important factor affecting students' physical health, its implementation condition is critical for the development of students' physical health. The following is the situation of physical education in the Garzê Tibetan schools.

\section{A. Low Utilization Rate of School Sports Grounds and Facilities}

The sports grounds and facilities in the primary and middle schools in Tibetan Autonomous Prefecture of Garzê are relatively complete. In terms of kinds of sports, the traditional Tibetan sports equipment and the basketball venues are more numerous, and the track and field sports grounds and equipment are relatively perfect. Although the venues and facilities in schools are relatively complete, the category is single, and there are fewer sports options, which affect the teachers' teaching initiative and the students' enthusiasm for sports. In addition, the use rate of sports facilities is relatively low, which is mainly reflected in the lack of "professionalism" of physical education classes, and the organization of extracurricular sports activities is not adequate, resulting in sports equipment and venues not being made the best use of. In addition, the high altitude and special climate of the Tibetan Autonomous Prefecture of Garzê lead to only one-third of the time each year suitable for outdoor activities ${ }^{6}$, but the number of indoor sports stadiums in primary and middle schools is very small, the sports activities are mainly carried out outdoors. In terms of the hardware, this is a very important constraint that restricts students in Tibetan Autonomous Prefecture of

Yin Defu. Investigation and analysis of physical fitness status and influencing factors of Tibetan primary and middle school students living in Tibetan Autonomous Prefecture of Garzê, Sichuan for generations[D]. Chengdu Sport Institute, 2017.

Yin Defu. Investigation and analysis of physical fitness and influencing factors of primary and middle school students in Tibetan Autonomous Prefecture of Garzê, Sichuan [D]. Chengdu Sport Institute. 2014 
Garzê from taking physical education classes and extracurricular sports activities.

\section{B. Lack of Physical Education Teachers}

The lack of physical education teachers is mainly manifested in two aspects: the total number of physical education teachers is small and the professional physical education teachers are not adequate. According to the standard of allocating one physical education teacher for every six classes in the compulsory education, the average vacancy rate of physical education teachers in Tibetan Autonomous Prefecture of Garzê is more than 30\%, and the vacancy rate of physical education teachers in some areas reaches nearly $60 \%$. The physical education teachers are seriously insufficient ${ }^{7}$. Apart from the growing number of students in the urban area leading to a shift in teacher-student ratios, the main reason for this phenomenon lies in two aspects: First, there are fewer local students who are admitted to foreign universities, causing that the prefecture is unable to achieve "self-production and self-sale". Second, foreign students are reluctant to go to Tibetan Autonomous Prefecture of Garzê for work. These two aspects are both the cause of the shortage of the physical education teachers in the region and the cause of the lack of professional physical education teachers. From the interviews, we learned that for the in-service physical education teachers, the factors affecting the instability of physical education teachers in the Garzê area are mainly that the professional titles and income problems are not solved, the opportunities for pursuing further education and learning are few, the objective conditions of teaching are too poor, and the work is hard. According to the degree of influence, the above factors are ranked first, second, third and fourth respectively. The lack of attention from relevant leaders and the low social status of physical education teachers are also important factors for the lack of physical education teachers in the region.

\section{Implantation of Exam-oriented Education}

Since the founding of the People's Republic, especially since the reform and opening up, the rapid development of China's economy has driven the development of various undertakings throughout the country. This is especially obvious in the education career. The Tibetan Autonomous Prefecture of Garzê is affected by the rapid development of education in the central and eastern regions, and more and more Tibetan migrant workers are going out to see the new scenes and the big world, which has led to the transformation of local concepts, greatly improved the rate of enrollment and promoted the development of local education. However, knowledge can arm the mind, but can not directly promote the physical health of students. With the increasing of demand for the enrolment rate in the examination-oriented education, the examination-oriented education is gradually affecting the development of physical fitness of the primary and middle school students in the Tibetan area. Devoting the power of the whole school into conducting examination preparation and

Yin Defu. Investigation and analysis of physical fitness and influencing factors of primary and middle school students in Tibetan Autonomous Prefecture of Garzê, Sichuan [D]. Chengdu Sport Institute. 2014. teaching is also the main reason for the lack of extracurricular sports activities.

\section{Physical Education Is Not Targeted and Has Poor Results}

For primary and middle school students living in the plateau and at the growing stage, the average altitude of 3,500 meters affects the development of their bodies. Their postures, heights, etc. are all affected by the high altitude and the special climate of the Tibetan Autonomous Prefecture of Garzê. In the school physical education in the plateau environment, physical exercise directly affects and promotes the speed and endurance In the process of teaching, how to conduct targeted teaching and training on students' speed and endurance according to their physique, and how to combine the special geographical and climatic conditions of the Garzê Tibetan area to set the curriculum, is a difficult task to accomplish. From the perspective of five evaluations of physical education, motor skills should still be an important part of physical education.

\section{E. Insufficient Development of Extracurricular Sports Activities}

The lack of extracurricular sports activities in the Garzê Tibetan primary and secondary schools is mainly reflected in the small number of sports and the small number of development times. In addition to the traditional Tibetan sports, basketball, track and field and table tennis are the main sports, and the frequency of development is 1 time/year. The football sport is carried out for relatively more times, which is only 1-2 times/year. Doing gymnastics and running are the main forms to conduct sports activities in major sport breaks, and there is no effective physical exercise monitoring guarantee. Compared with the requirements of China for primary and secondary schools of "arranging three or more times of extracurricular sports activities per week, and ensuring that students have one hour of physical activity per day (including physical education classes)", the physical exercise time of primary and middle school students in the Garzê Tibetan area is not guaranteed. Sports time is the foundation of physical health. It can be seen that the physical health of primary and secondary school students in the area cannot be guaranteed from the aspect of exercise. ${ }^{8}$

\section{CONCLUSION}

There are many factors influencing the physical health of Garzê Tibetan primary and middle school students. From the perspective of the great education, in addition to responding to and implementing national education policies, education poverty alleviation policies, and student physical health monitoring standards, social education, family education, and school education are all very important and can have a direct impact on the promotion of physical health of primary and secondary school students in the Garzê Tibetan area. School physical education as an important part of school education, its development is crucial to the physical health of primary and

\footnotetext{
$8 \quad$ Zhao Wentao. Research on the current situation and countermeasures of physical education in primary and middle schools in Tibetan Autonomous Prefecture of Garzê, Sichuan [D]. Chengdu Sport
} Institute, 2012 
secondary school students in Garzê Tibetan area. As Professor $\mathrm{Yu}$ Sumei, a researcher at the Institute of Sports Health Arts Education of National Institute of Education Sciences, said, the current development of students' physique should be focused on what can be acquired through efforts and can have direct impact on the physique of students. Mainly summarized as "one body and two wings", one body means school physical education, the two wings are: the left wing for nutrition, the right wing for sleep.

\section{A. One Body and Two Wings, One Body Being the Core}

The influence of school physical education on students' physical fitness is very important. In terms of duration, among the four components of school physical education, the proportion of time of physical education classes and sports major breaks ranks the first and second respectively. While doing a good job in sports skill learning in physical education classes, the schools should arrange sports activities in the afterschool hours (such as inter-class exercises, sports major breaks, and conducting sports club activities in the afternoon) combining with the fields and layouts of the schools to ensure the daily sports time of students not less than an hour. Combining the altitude characteristics of the Garzê Tibetan area, focus on training the students' speed and endurance. In addition, physical exercise will also be affected by the atmosphere. In the context of the school, organizing sports competitions as an opportunity to create a good physical exercise atmosphere, can promote the active participation of primary and secondary school students in physical exercise, thus promoting the development of students' physical fitness.

\section{B. One Body and Two Wings, Nutrition Being the Foundation}

Under the premise that the innate genetic factors cannot be changed, nutrition is the foundation to promote the physical health of students. During the field trip, we found that although the Garzê Tibetan primary and secondary school students were immersed in the spring breeze of "two exemptions and one subsidy", only the problem of eating and clothing was solved. The dishes were too single, not to mention the nutritional balance. Therefore, it is also necessary to hire and select chefs with certain nutritional knowledge. The local department should provide subsidy policies that match the "two exemptions and one subsidy" policy, and control the purchase of food ingredients to properly arrange the dishes and nutrition. ${ }^{9}$

\section{One Body and Two Wings, Sleep being a Guarantee}

Du Faqiang mentioned in the analysis of the causes of physique health of adolescents in China that compared with primary and middle school students who have slept for more than 7 hours, the primary and secondary school students who sleep less than 7 hours or even less than 6 hours have adolescent body health rating tends of 1.910 times and 1.978

\footnotetext{
9 Zhao Wentao. Research on the current situation and countermeasures of physical education in primary and middle schools in Garz ê Tibetan Area, Sichuan Province [D]. Chengdu Sport Institute, 2012.
}

times respectively ${ }^{10}$. In addition, under the premise of ensuring sleep time, sleep quality is an important factor that has a direct impact on the physical health of students. High-quality sleep can not only promote the secretion of growth hormone in the body, but also reduce the incidence of obesity. With the accelerated pace of life, the widespread use of smart technology products has more and more impacts on the Tibetan primary and secondary school students in Garzê, and their sleep time and sleep quality should be paid more attention to. The education authorities and schools should strengthen the management of students' work and rest, first of all to ensure that the sleep time of Tibetan primary and middle school students in Garzê is about 8 hours.

\section{Accurate Support, Realizing Seamless Connection between Research and Teaching}

The core of the training of talents is education, the core of education lies in the teachers, to solve the problem of lack of physical education teachers in the Garzê Tibetan area and the unprofessional and not targeted physical education, to introduce high-quality physical education professionals, and to provide after-service training and physical education professional talents accurate partner support and training for in-service physical education teachers is a good way. Carry out horizontal cooperation (school-school cooperation, schoollocal cooperation) and other means to introduce and re-train talents. At the same time, carry out the combination of research and first-line teaching, the combination of the physical education theories and practice, and cultivate physical education talents with professional qualities to effectively promote the development of physical fitness of primary and secondary school students in Tibetan Autonomous Prefecture of Garzê.

\section{REFERENCES}

[1] Yu Sumei.The Construction of the Core Literacy Framework System of Chinese Students' Physical Education Discipline[J].Journal of Physical Education,2017,24(04):pp.5-9.

[2] Bama amo. The status quo, dilemma and breakthrough of education development in Tibetan areas in the past ten years-Taking Tibetan Autonomous Prefecture of Garzê as an example[J]. Journal of Southwest University, Social Science Edition, 2012, 1001:pp.172-176.

[3] Yu Sumei.Basic Thoughts and Multiple Ways of Cultivating the Core Literacy of Students' Physical Education[J].Journal of Physical Education,2017,24(05):pp.16-19.

[4] Current Situation and Thinking of Education in the Garzê Tibetan Area[J]. Ye Xiaobin. Journal of Southwest Jiaotong University (Social Science Edition). 2010(04)

[5] Liu Huibin, SONG Yi, MA Jun, ZHANG Bing, HU Peijin. Analysis of Dynamic Trends and Changes of Speed and Endurance Quality Index of Tibetan Primary and Middle School Students in Tibet from 1985 to 2010[J]. Chinese Journal of Child Health Care, 2016, 24(07): pp.686 $690+694$.

[6] Chen Bo. Research on the Status Quo and Problems of Physical Education Teachers in Compulsory Education Stage in Garzê Tibetan Area[J].Journal of Sichuan Minzu College,2011,20(01)

10 Du Faqiang, Fan Jingjing. Analysis of the Causes of Physical Health of Juvenile Students in China [J].Sports and Science, 2014, 35(03): pp.60-67. 
[7] Ye Xiaobin.The Status Quo and Thinking of Education in Garzê Tibetan Area[J].Journal of Southwest Jiaotong University(Social Science Edition),2010,11(04):pp.94-99.

[8] Lin Junhua. An Introduction to the History of School Education Development in Garzê Tibetan Area[J]. Tibetan Studies, 1993(02): pp.39-46+17. 